\title{
Complexity ratings of digit strings and their pictorial analogs*
}

\author{
DOUGLAS W. OHMAN and PAUL C. VITZ \\ New York University, New York, N.Y. 10003
}

In each of three experiments, one group of Ss rated complexity of short strings of decimal digits and another group rated the complexity of the pictorial analogs of the digit strings. The pictorial analog of a digit string was the dot-line graph or bar graph representing the heights of the digits in the string. The physical properties of these two types of stimuli are quite different, but the mean complexity ratings for 2-, 5- and 8-place digits and their pictorial analogs had high positive correlations. This was interpreted as evidence that the underlying code for the two types of stimuli is similar. Some evidence for the effect of digit size and size of digit differences on complexity ratings was also found.

In one condition of the following experiments, Ss are shown examples of strings of decimal digits typed on a card and asked to rate the complexity of the string using a 7-point scale. In the other condition, different Ss rate the complexity of a pictorial analog of each of the digit strings, e.g., the dot-line graph or bar graph which can be constructed from the digit string. See Fig. 1 for examples of the pictorial analogs. Digit strings and their pictorial analogs are obviously quite different in their physical properites; however, we hypothesize that the complexity ratings of these two types of stimuli will have a high positive correlation, and that such a correlation implies that the codes for these stimuli are very similar.

There are several models of the coding of event strings, e.g., Simon \& Kotovsky (1963), Vitz \& Todd (1969), and Restle (1970). None of these systems specifies how $\mathrm{S}$ codes short arbitrary strings of digits, but, given the assumption $S$ repeatedly scans the string from left to right, making it a cyclic pattern, Restle's theory is applicable to the pictorial analogs of such strings, and support for the hypothesis also implies that Restle's theory is applicable to digit strings.

When the hypothesis was suggested to several colleagues, they expressed doubt about its validity. The commonest reason for skepticism was the belief that, whatever the code for strings of digits, it seemed unlikely that it would be similar to the code for such perceptual stimuli as graphs and, hence, that the complexity ratings of the two stimulus types should not be correlated. For example, the digits might be coded as a string of digit names and the complexity of this word string presumably would have little relation to the complexity of the verbal or other type of code used for the graphs.

The complexity rating task was chosen, since ratings

* Reprint requests should be sent to P. C. Vitz, Department of Psychology, New York University, 4 Washington Place, New York, N.Y. 10003. This research was supported by Grant NS-07636-04AL from The National Institutes of Health to the second author. can be gotien quickly and reliably on a large number of strings and because complexity ratings of strings have been shown to be highly related to such dependent variables as learning difficulty, perceptual and short-term memory difficulty, number of words used to describe the string, etc. (Vitz \& Todd, $1969^{1}$ ).

\section{METHOD}

Stimuli

Experiment 1A: One hundred 8-place decimal numbers, shown in Table 1, were typed on $4 \times 6$ cards; 67 were selected on the basis of E's judgment to account for the major types of pattern and structure in digit strings, e.g., runs, alternations; the remainder were picked randomly. Experiment 1B: Each number of Experiment $1 \mathrm{~A}$ was converted into a dot-line graph analog (see Fig. 1). The dots were $1 / 2$ in. apart; vertical distance was in 1/4-in. steps. Experiment 1C: Each number of Experiment 1A was converted into a bar graph (histogram) analog (see Fig. 1). The bars were $5 / 8 \mathrm{in}$. wide, the steps, $1 / 4 \mathrm{in}$. high. The graphs were drawn in ink on a $4 \times 6$ card. The main difference between the two analogs is in their treatment of zero, which is a dot on the $\mathrm{x}$-axis (dot-line graph) or a blank space (bar graph). Experiment 2A: Ninety-nine decimal numbers typed on a card: 32 2-place numbers, 33 5-place, and 34 8-place. These are shown in Table 2. (The failure to have 100 numbers was an oversight.) About two-thirds of each set of numbers were selected to cover the major kinds of pattern and structure-the rest were selected randomly. Experiment 2B: The dot-line analogs of the 99 numbers. Experiment 3A: Fifty 2-place digits, selected at random, from the range of 10-99; 50 5-place digits, selected at random, from the range of 10,000-99,999; all were typed on cards. Experiment 3B: The bar-graph analogs of the Experiment $3 \mathrm{~A}$ digit strings drawn on cards.

\section{Procedure and Subjects}

The $S$ rated complexity on a 7-point rating scale with 1.0 being very simple and 7.0 being very complex. Stimulus cards were presented in a different random order to each $S$. If $S$ raised a question about what complexity meant, he was told to think of simple stimuli as easy to remember or memorize and complex stimuli as difficult. After Experiments 1A and 2A, S was asked if he had thought of the stimulus as a graph or profile; after Experiments 1B, 1C, and 2B, S was asked if he had thought of the stimulus as a string of digits; Ss in Experiment $1 \mathrm{C}$ were also asked what properties characterized simple and complex patterns. Twelve students were $\mathrm{Ss}$ in each experiment.

\section{RESULTS AND DISCUSSION \\ Experiments 1A, B, C}

The correlation between the mean complexity of the digit strings and the mean dot graph complexity is .84 : between digit string complexity and bar graph complexity, .91 ; between the two analog measures, .86 . The rank order of the mean complexity ratings from lowest to highest of the digit strings in Experiment $1 \mathrm{~A}$ is shown in Table $1 .{ }^{2}$ After the analog experiments, 1B and $1 \mathrm{C}$, no $\mathrm{S}$ reported thinking of the graph stimuli as a string of digits, i.e., as a string of absolute values. But six Ss in Experiment $1 \mathrm{~A}$ said they sometimes thought of the digit strings in some analog fashion, e.g., a series of "ups and downs." After Experiment 1C, Ss described simple bar graph patterns with expressions like: "completely 


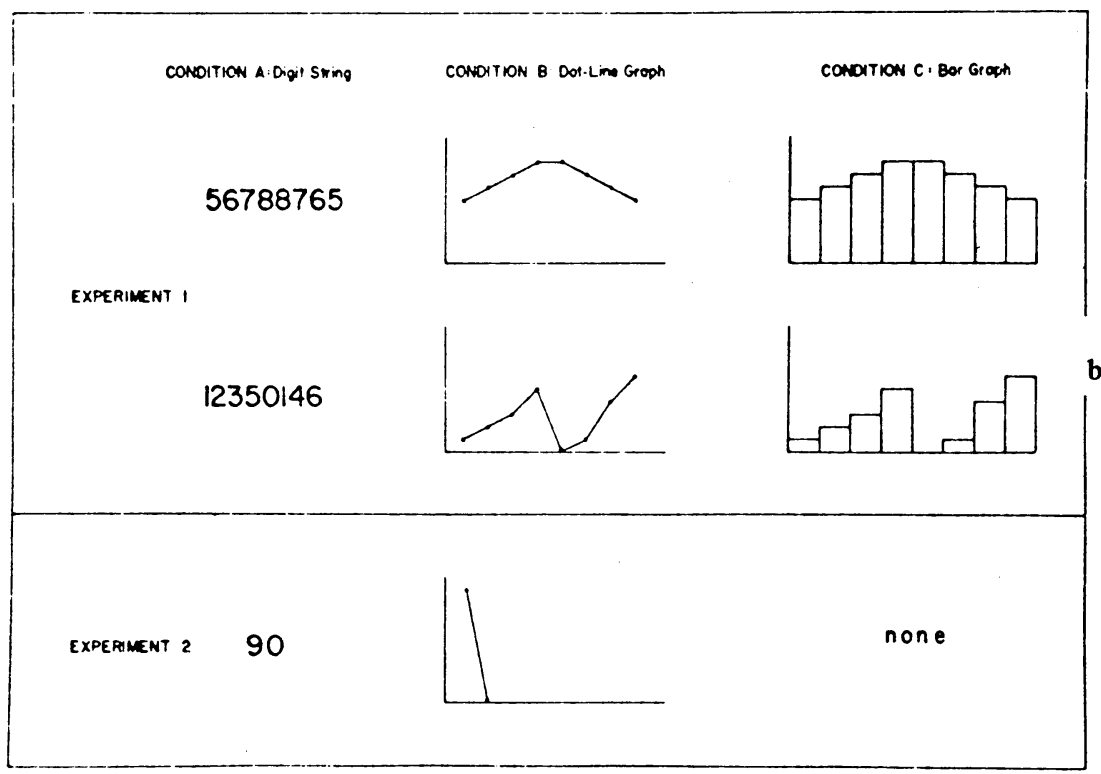

Fig. 1, Examples of dot-line graph and bar-graph pictoral analogs of digit strings.

the same height," "regularly steps up and down," etc. Such comments refer to elements, such as runs or alternations, commonly proposed by various theorists to describe sequential structure. Together, the results represent support for the hypothesis of a similar underlying code for the two stimulus types, and that Restle's coding system is probably applicable to short digit strings.

The two most discrepant points from the regression line of Experiment $1 \mathrm{~A}$ vs Experiment $1 \mathrm{~B}$ were Stimuli 33 and 63 . The ratings of these strings were bimodal-Ss with low ratings who apparently noticed the pattern and others with high ratings who did not. Often Ss made very rapid ratings, and some failed to detect patterns which were not immediately obvious; hence, if the task required greater attention to the digits, the correlation might be still higher. Certain stimuli, e.g., 19, 26, and 39 , which had higher dot-line ratings than digit ratings, consisted of large differences in the height of adjacent dots. If so, large adjacent differences apparently contribute more to complexity in a graph pattern than in a digit string. The scatter diagram of the correlation between Experiments $1 \mathrm{~A}$ and $\mathrm{C}$ was similar to $1 \mathrm{~A}-\mathrm{B}$.

There was a small tendency for 14141414 and 1234567 to be rated less complex than the same digits in the reverse order. This possible digit order effect was not

Table 1

The 100 Digit Strings of Experiment 1A Listed in Order of Their Mean Complexity Rating from Lowest to Highest

\begin{tabular}{|c|c|c|c|c|c|c|c|}
\hline No. & Stimulus & No. & Stimulus & No. & Stimulus & No. & Stimulus \\
\hline 1 & 00000000 & 26 & 28232823 & 51 & 38552644 & 76 & 21137491 \\
\hline 2 & 61111111 & 27 & 10110100 & 52 & 87732264 & 77 & 06155724 \\
\hline 3 & 12121212 & 28 & 13572468 & 53 & 56095206 & 78 & 94638875 \\
\hline 4 & 14141414 & 29 & 00771159 & 54 & 31539008 & 79 & 35742806 \\
\hline 5 & 19191919 & 30 & 22227493 & 55 & 00631972 & 80 & 14723809 \\
\hline 6 & 41414141 & 31 & 15523456 & 56 & 07868891 & 81 & 30624259 \\
\hline 7 & 54545454 & 32 & 95999828 & 57 & 69645803 & 82 & 49423972 \\
\hline 8 & 55557777 & 33 & 56788765 & 58 & 38075514 & 83 & 13674928 \\
\hline 9 & 68666666 & 34 & 97531444 & 59 & 62480180 & 84 & 76314820 \\
\hline 10 & 44446444 & 35 & 50007114 & 60 & 10792291 & 85 & 85960718 \\
\hline 11 & 12312312 & 36 & 36243600 & 61 & 60317688 & 86 & 93824160 \\
\hline 12 & 11181111 & 37 & 56638999 & 62 & 29808397 & 87 & 25798621 \\
\hline 13 & 12345678 & 38 & 59172233 & 63 & 21436587 & 88 & 28491731 \\
\hline 14 & 40666666 & 39 & 90271100 & 64 & 67450321 & 89 & 85493621 \\
\hline 15 & 33333833 & 40 & 85319777 & 65 & 37198805 & 90 & 18362749 \\
\hline 16 & 99887766 & 41 & 17204411 & 66 & 52866413 & 91 & 69710284 \\
\hline 17 & 88898888 & 42 & 36662948 & 67 & 97849197 & 92 & 63514091 \\
\hline 18 & 41741741 & 43 & 57300299 & 68 & 37318652 & 93 & 49381652 \\
\hline 19 & 80808020 & 44 & 12350146 & 69 & 84727669 & 94 & 72193604 \\
\hline 20 & 00010003 & 45 & 74666931 & 70 & 70675092 & 95 & 06389514 \\
\hline 21 & 32132132 & 46 & 92226481 & 71 & 58319962 & 96 & 61428213 \\
\hline 22 & 87654321 & 47 & 55751189 & 72 & 58103726 & 97 & 84017536 \\
\hline 23 & 96969496 & 48 & 51388864 & 73 & 49756883 & 98 & 92657103 \\
\hline 24 & 56655665 & 49 & 08908066 & 74 & 82591146 & 99 & 71865794 \\
\hline 25 & 66866666 & 50 & 71614101 & 75 & 21165738 & 100 & 76314259 \\
\hline
\end{tabular}


Table 2

The 99 Digit Strings of Experiment 2A (the Two-, Five-, and Eight-Place Strings are Listed in Order of Their Mean Complexity Rating from Lowest to Highest

\begin{tabular}{|c|c|c|c|c|c|c|c|}
\hline No. & Stimulus & No. & Stimulus & No. & Stimulus & No. & Stimulus \\
\hline 1 & 10 & 26 & 26 & 51 & 67686 & 76 & 81444479 \\
\hline 2 & 15 & 27 & 56 & 52 & 04979 & 77 & 75111138 \\
\hline 3 & 30 & 28 & 93 & 53 & 66375 & 78 & 11117583 \\
\hline 4 & 88 & 29 & 58 & 54 & 11873 & 79 & 11117538 \\
\hline 5 & 14 & 30 & 89 & 55 & 81137 & 80 & 07663432 \\
\hline 6 & 12 & 31 & 82 & 56 & 45367 & 81 & 35799753 \\
\hline 7 & 11 & 32 & 78 & 57 & 93905 & 82 & 83050921 \\
\hline 8 & 77 & 33 & 12345 & 58 & 83711 & 83 & 92788338 \\
\hline 9 & 99 & 34 & 22333 & 59 & 83117 & 84 & 76710313 \\
\hline 10 & 22 & 35 & 28282 & 60 & 34117 & 85 & 96341565 \\
\hline 11 & 44 & 36 & 12121 & 61 & 72419 & 86 & 73212819 \\
\hline 12 & 80 & 37 & 19191 & 62 & 17935 & 87 & 11725483 \\
\hline 13 & 23 & 38 & 12221 & 63 & 23761 & 88 & 62130054 \\
\hline 14 & 45 & 39 & 23232 & 64 & 87281 & 89 & 36798344 \\
\hline 15 & 60 & 40 & 54321 & 65 & 52594 & 90 & 41396545 \\
\hline 16 & 70 & 41 & 77888 & 66 & 11111111 & 91 & 93682722 \\
\hline 17 & 90 & 42 & 87654 & 67 & 99999999 & 92 & 26543178 \\
\hline 18 & 34 & 43 & 89998 & 68 & 55555555 & 93 & 50798344 \\
\hline 19 & 49 & 44 & 12131 & 69 & 12344321 & 94 & 17215934 \\
\hline 20 & 76 & 45 & 89898 & 70 & 99663311 & 95 & 93622827 \\
\hline 21 & 65 & 46 & 45678 & 71 & 11336699 & 96 & 72511483 \\
\hline 22 & 67 & 47 & 78787 & 72 & 24624624 & 97 & 38539407 \\
\hline 23 & 97 & 48 & 44220 & 73 & 64264264 & 98 & 72548311 \\
\hline 24 & 55 & 49 & 23242 & 74 & 44448179 & 99 & 22936827 \\
\hline 25 & 39 & 50 & 88664 & 75 & 81794444 & & \\
\hline
\end{tabular}

statistically significant, but it occurred in all three experiments. If reliable, this effect may be due to greater familiarity with the forward order or, assuming left to right scanning, to a primacy effect in which the larger element is weighted more heavily when it occurs first. There is also a suggestion, in the ordering of the means of Numbers 3, 4, and 5 in Experiment $1 \mathrm{~A}$, that if two patterns are structurally identical, then the one with the larger digits is rated more complex. This is not so in the analog pattern data.

\section{Experiment 2A-B}

These experiments were conducted to test the hypothesis on 2- and 5-place digits, to replicate the results on 8-place digits, and to investigate further the digit order effect and the possible effect of digit size and size of digit differences in structurally identical patterns.

The correlation between the mean ratings of the digit strings and their analogs is .32 for 2-place digits, .87 for the 5-place digits, and .88 for the 8-place digits. Table 2 presents the digit strings in the rank order of mean complexity in Experiment 2A. The last two correlations extend and replicate the results of Experiment 1. The low correlation for the 2-digit stimuli presumably occurred because the dot-line graph ratings differed very little and there is little variance to be explained and because divisibility by 10 significantly lowers digit string ratings, while this property has no special existence in the dot-line pictorial representation. Again, Ss that rated the analog stimuli did not report thinking of the graphs as strings of digits, but Ss rating the digit strings frequently reported coding part or all of the string into some kind of graphical representation.
In Experiment 2A, 12345 and 24624624 are judged simpler than their reverse order but 45678 is rated slightly more complex than 87654 , and hence evidence for a normal digit order effect remains weak. (None of the differences are statistically significant.) In Experiment $2 \mathrm{~A}$, there is evidence that in structurally identical patterns the pattern with the larger digits is rated more complex. In all of the following pairs, the larger digit string is rated more complex: 89998 vs $12221 ; 77888$ vs $22333 ; 67686$ vs $23242 ; 23242$ vs $12131 ; 88664$ vs 44220 . One small exception is that 78787 is slightly more complex than 89898 . Excluding tied ratings, the mean number of higher ratings for the "large" member of the pair was 3.0 and 1.33 for the smaller $(t=3.01, d f=10, p<.05$, two-tailed $)$. This same pattern of results does not reliably characterize the ratings for the analog stimuli, since apparently, within the framework of these small scale graphs, the absolute height had no significance. There is again weak evidence that large successive differences contribute to the complexity of the graph patterns: 19191 is slightly more complex than 23232 (not statistically significant).

These modest "size" effects might have been greater if the task had required $S$ to compare the relevant stimuli directly. Regardless, such effects are consistent with the results of Moyer \& Laudauer (1967) on the effect of digit size and digit difference on reaction time, and with evidence that of two structurally identical numerical progressions the one constructed from larger digits takes longer to learn. ${ }^{1}$

\section{Experiment 3A-B}

These experiments provide an esitmate of the 
correlation on randomly drawn 2- and 5-place stimuli. Also. using the bar graph representation which had the higher $\mathrm{r}$ in Experiment 1 allows for a new test of the hypothesis on 2-digit numbers. The correlation for the 2-digit stimuli between mean digit string complexity and mean bar graph complexity is .77 , a substantial improvement over .32 , which presumably occurs because the bar graph analog had a blank space for zero instead of a dot. The corresponding correlation for the 5-place stimuli is .55, which is lower than in Experiment 2 and implies that for randomly selected stimuli, longer than 2 places, the correlation is much lower than for such samples as in Experiments 1 and 2. The 5-place strings in the random sample were much more similar and their ratings had a much smaller range than those in Experiment 2, and as a result, the between-S rating agreement dropped from a median $\mathrm{r}$ of .77 in Experiment 2 to .36 . Very likely, the $r$ for random samples declines progressively as string length increases.

If it can be assumed that rated complexity of digit strings is a reasonably good estimate of processing difficulty, e.g., memory difficulty, then the results of the three experiments imply that any model of the processing of digit strings must take into account the ease of coding or structurally organizing the string, and even possibly the "size" effects. Such properties as the number of digits and their location in the string are presumably not sufficient.

The mean complexity ratings of the 502 -digit strings and their bar graph analogs were each correlated with the association values of 2 -digit numbers provided by Battig \& Spera (1962). The $r$ values were -.659 and -.343 , respectively. This suggests that at least part of the association value of a number is due to its structural complexity.

\section{REFERENCES}

Battig, W. F., \& Spera, A. J. Rated association value of numbers from 0-100. Journal of Verbal Learning \& Verbal Behavior, $1962,1,200-202$.

Moyer, R. S. \& Landauer, T. K. Time required for judgments of numerical inequality. Nature, 1967, 215, 1519-1520.

Restle, F. Theory of serial pattern learning: Structural trees. Psychological Review, 1970, 77, 481-495

Simon, H. A., \& Kotovsky, K. Human acquisition of concepts for sequential patterns. Psychological Review, 1963, 70, 534-546.

Vitz, P. C., \& Todd, T. C. A coded element model of the perceptual processing of sequential stimili. Psychological Review, 1969, 76, 433-449.

\section{NOTES}

1. Vitz, P. C., \& Hazan, D. Learning numerical progressions. Unpublished manuscript, 1973.

2. The mean complexity ratings of the stimuli in Experiments $1 \mathrm{~A}-\mathrm{B}-\mathrm{C} ; 2 \mathrm{~A}-\mathrm{B} ; 3 \mathrm{~A}-\mathrm{B}$ are available from the second author.

(Received for publication A pril 18, 1973.) 